\title{
Wireless RAN and Frequency Hopping Schemes in Cognitive Radio Network, An Overview
}

\author{
Sonali Gupta ${ }^{1}$, Sonam Rathore ${ }^{2}$ \\ Assistant Professor, Electronics \& Communication Department, Shri Ram Murti Smarak College of Engineering \\ Technology \& Research, Bareilly ${ }^{1,2}$
}

\begin{abstract}
The IEEE 802.22 WRAN is the first standard which is based on the cognitive radio network. WRAN basically uses unused or white spaces within the television bands between 54 and $862 \mathrm{MHz}$, especially within rural areas where usage may be lower. One of the major challenges for WRAN is how to efficiently schedule both the spectrum sensing and data transmission simultaneously. This problem for signal transmission is termed as coexistence. In this paper we give an overview of WRAN and discuss some techniques to reduce coexistence.
\end{abstract}

Keywords: Cognitive radio Network (CRN), white space, spectrum sensing, coexistence.

\section{INTRODUCTION}

IEEE 802.22 WRAN system is supposed to be the first wireless communication standard using cognitive radio functionalities [1]. The IEEE 802.22 standard defines a system for a Wireless Regional Area Network, WRAN that uses unused or white spaces within the television bands between 54 and $862 \mathrm{MHz}$ in most of the cases within rural areas where usage of frequency spectrum may be lower[2]. In November/2004, the first air interface PHY as well as MAC standard based on Cognitive Radios (CRs) was developed, which known as the IEEE 8.2.22 Working Group (WG)[3]. The IEEE 802.22 WG is included in the development of a CR-based Wireless Regional Area Network (WRAN) [3].with WRAN some of the first cognitive networks could be deployed [2].

There are a number of points to be considered for the better understanding of the basis of the 802.22 standard. These include terms such as the system topology, system capacity and the projected coverage for the system.

\section{A. System topology}

The system is intended to be a point to multipoint system, i.e. it has a base station with a number of users or Customer Premises Equipments, CPEs located within a cell.

\section{B. Coverage area}

The coverage area for the IEEE 802.22 standard is much greater in comparison to many other IEEE 802 standards.

For 802.22, the specified range for a Central Premise Equipment (CPE) is approximated to be $33 \mathrm{~km}$ and for some cases base station coverage may extend to $100 \mathrm{~km}$. For the coverage area of $33 \mathrm{~km}$, the power level of the CPE is $4 \mathrm{Watts}$ EIRP (effective radiated power relative to an isotropic source).

\section{System capacity}

The downlink or download speed of system at the cell periphery is about $1.5 \mathrm{Mbps}$ and that of an uplink or upstream speed is $384 \mathrm{Kbps}$. For this downlink and uplink system capacity 12 simultaneous users are assumed in cognitive radio network. To achieve this the overall system capacity must be $18 \mathrm{Mpbs}$ in the downlink direction [2].The functional requirements for IEEE 802.22 WRAN are as follows:

Functional requirement

- General

\begin{tabular}{|l|l|}
\hline Parameter & Requirement \\
\hline Carrier frequency & UHF, VHF band $<1 \mathrm{GHz} 54 \sim 862 \mathrm{MHz}$ \\
\hline & $6(7,8) \mathrm{MHz}$ \\
Bandwidth & It may be a smaller band or a combination of multiple bands \\
\hline & Fixed location customers \\
& - Residential \\
Service subscribers & - Small and Medium Enterprises \\
\hline
\end{tabular}




\begin{tabular}{|l|l|}
\hline Type of Service & Packet service (data, voice or vedio) \\
\hline Service model & $\begin{array}{l}\text { Same as ADSL \& cable MODEM service model of wireless regional } \\
\text { area network (WRAN) used in less populated rural areas. }\end{array}$ \\
\hline
\end{tabular}

- $\quad$ Target Performance

\begin{tabular}{|l|l|}
\hline Parameter & Requirement \\
\hline Number of active subscriber & Minimum 12 users in CRN \\
\hline Service Coverage & Minimum- 33km, Maximum- $100 \mathrm{Km}$ \\
\hline Minimum peak Throughput at cell egde & Uplink- $1.5 \mathrm{Mbps}$, Downlink- $384 \mathrm{Kbps}$ \\
\hline Spectral efficiency & Minimum- $0.5 \mathrm{bps}$, Maximum- $3 \mathrm{bps}$ \\
\hline Service availability & $50 \%$ of locations and $99.9 \%$ of time \\
\hline
\end{tabular}

- System Parameters

\begin{tabular}{|c|c|c|}
\hline Parameters & Specification & Remarks \\
\hline Frequency range & 54 to $862 \mathrm{MHz}$ & \\
\hline Bandwidth & $6,7,8 \mathrm{MHz}$ & $\begin{array}{l}\text { Channels can be used as aggregation of } \\
\text { discontigious channel or bonding of upto } 3 \\
\text { contigious tv channel can be utilized. }\end{array}$ \\
\hline Service coverage & $33 \mathrm{Km}$ & \\
\hline Data rate & $\begin{array}{l}\text { Maximum- 23 Mbps } \\
\text { Minimum- } 4.8 \mathrm{Mbps}\end{array}$ & Maximum of 72 can be use for 7 and $8 \mathrm{MHz}$ \\
\hline Modulation & QPSK, 16 QAM, 64 QAM & These modulation schemes are mandatory \\
\hline Spectral Efficiency & $\begin{array}{l}\text { Maximum- } 4.03 \mathrm{bits} / \mathrm{s} / \mathrm{Hz} \\
\text { Minimum- } 0.81 \mathrm{bits} / \mathrm{s} / \mathrm{Hz}\end{array}$ & \\
\hline Transmit power & 4 Watt & $\begin{array}{l}\text { Default effective radiated power to an } \\
\text { isotropic source }\end{array}$ \\
\hline Multiple Access & $\begin{array}{l}\text { Orthogonal frequency division multiple } \\
\text { access (OFDMA) }\end{array}$ & \\
\hline Duplex & Time Division Duplexing (TDD) & $\begin{array}{l}\text { Frequency Division Duplexing (FDD) is } \\
\text { also supported }\end{array}$ \\
\hline Cyclic Prefix Mode & $1 / 4,1 / 8,1 / 16,1 / 32$ & \\
\hline Network topology & Point to multipoint network topology & \\
\hline
\end{tabular}

\section{THE 802.22 AIR INTERFACE}

\section{A. The PHY Layer ( physical layer)}

The PHY layer must be adaptable to different conditions and also flexibility is required for jumping from channel to channel without errors in transmission or losing clients (CPEs)[5].The PHY layer offers high performance while keeping low complexity level[3]. An orthogonal frequency division multiplexing (OFDM) scheme has been adopted because the 802.22 WRAN system provide resilience against multipath propagation and selective fading as well as a high level of spectrum efficiency [2]. With OFDMA it will be possible to achieve this fast adaptation required for the BS's and CPEs. OFDMA will be the modulation scheme for transmission in uplink and downlink design[5].

B. The MAC Layer(medium access control)

We will consider two structures in the MAC layer design: Frame and Superframe. A superframe will be formed by many frames. The superframe will have an SCH (Superframe Control Header) and a preamble. These will be sent by the BS in every channel so that it's possible to transmit and not cause interference. When a CPE is turned on, it will continuously sense the spectrum and will find out which channels are available and will receive all the needed information to attach to the BS [5].

\section{COEXISTENCE}

As we discussed earlier that, IEEE 802.22 is the first wireless standard based on cognitive radio (CR) technology.CR technology enables unlicensed users in WRANs to utilize licensed (incumbent) spectrum bands on a non-interference basis to incumbent users. The coexistence between incumbent users and unlicensed users is referred to as incumbent 
coexistence. On the other hand, the coexistence between unlicensed users in different WRAN cells is referred to as self-coexistence [6].

One of the major challenges for WRANs is how To Efficiently scheduling of channel sensing and data transmission simultaneously for multiple adjacent WRAN cells is one of the major challenges for WRANs. There are four scheme that can reduce coexistence in IEEE 802.22 WRAN. These schemes are based on a well-known operation mode of IEEE 802.22, namely[7]

- Dynamic Frequency Hopping (DFH).

- $\quad$ Fixed-Scheduling DFH (FDFH)

- Cooperative DFH (CDFH)

- $\quad$ Sectoral DFH (SDFH)

- Fixed-Scheduling Sectoral DFH (FSDFH)

A. Dynamic Frequency Hopping (DFH)

An alternative operation mode called dynamic frequency hopping (DFH) is proposed in IEEE 802.22 WRAN, where WRAN data transmission is performed along with the spectrum sensing in the air interface without interruptions. DFH is a mechanism that coordinates multiple WRAN cells operating in the DFH mode, so that reliable channel sensing and efficient frequency usage are achieved [8].

A WRAN system in the DFH mode is shown in Figure 3. A WRAN system dynamically selects one of the channel which was validated in a previous operation of data transmission in the next operation period. This channel can be used for data transmission for up to two seconds which is the maximum channel detection time [8].

The FDFH and CDFH are two categories based on using omni-directional antennas at the base stations (BSs), whereas the BSs in the SDFH and FSDFH uses directional antennas [7].

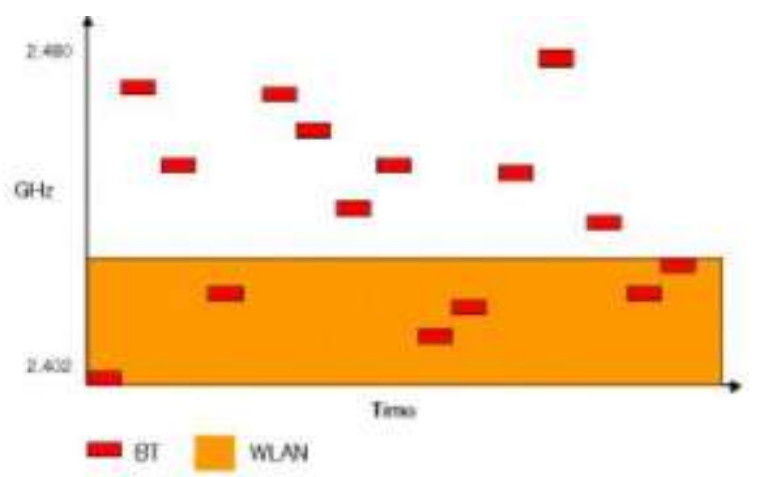

Fig.1: Dynamic Frequency Hopping Operation

B. Fixed-scheduling DFH (FDFH)

In FDFH Neighboring WRAN cells determine a fixed schedule for selecting the next working channel [9]. To understand FDFH scheme consider a WRAN system with three BSs:

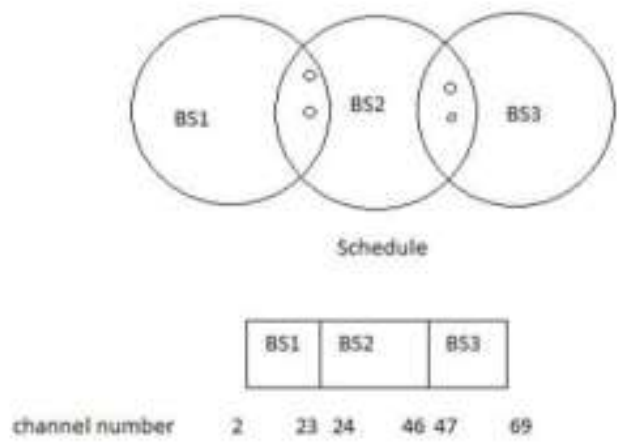

Fig.2: Example illustrating the FDFH scheme (67 TV channels used in USA).

In this given figure BS1 selects its next working channel among channels 2 to 23 , starting from channel 2. If BS1 finds an available channel in this range, it uses this channel as its next working channel. Otherwise, it will search for an available channel in the range of channels that are given to BS3, i.e., channels 47 to 69. If BS1 still does not find an available 
channel than it will search in the range of channels that are given to BS2, i.e., channels 24 to 46 (starting from channel 46 to reduce the chances of selecting the same channel that BS2 may choose). BS2 and BS3 apply the same procedure [7].

\section{Cooperative DFH (CDFH)}

A more flexible scheme is proposed, namely Cooperative DFH. The key idea of CDFH is that each WRAN cell cooperatively selects its working channels, taking into account the working channels of its neighboring cells. The main features of the CDFH scheme, which are: listening and sensing, and channels selection and Synchronization.

The CDFH scheme requires synchronization among adjacent BSs. The purpose of listening process in CDFH is to detect all the beacons that have been sent by adjacent BSs. Listening process is different from the sensing process in CDFH. The purpose of the sensing process is to determine the available channels that are not occupied by primary users.

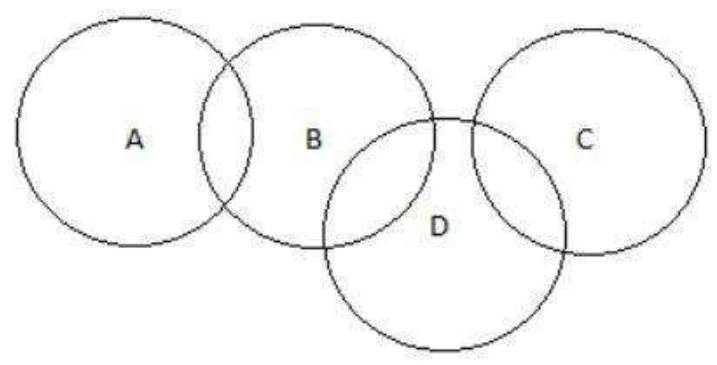

Fig.3: synchronization between BSs in $\mathrm{CDFH}$.

Based on the results of the listening process, the BS can use the channels that are not occupied by its adjacent BSs that have common users (in the overlapped area) with this BS. According to the proposed CDFH scheme, a BS has the ability to reuse the channels that are occupied by its adjacent BSs [7].

\section{Sectoral DFH (SDFH)}

The CDFH scheme require an overhead coordination between the base stations. This problem is overcome in SDFH. The SDFH scheme is free from overhead coordination. The SDFH divides the WRAN into sectors, which helps to reduce the overlapping between neighboring BSs [9].

According to the sectoring feature of SDFH, nodes B and D lie in an overlapped sector between $\mathrm{X}$ and $\mathrm{Y}$, and they are served by the same channel (channel 2). Thus, none of them is served. Although, nodes A, C and E are served, as they lie in sectors that do not use the same channels for both BSs [7].

SDFH results in more efficient as more number of users are served in the overlapped areas between the neighboring BSs.

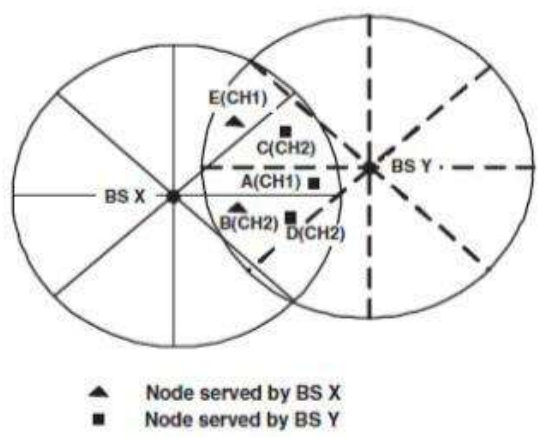

Fig. 4: Example illustrating the SDFH scheme.

E. Fixed-scheduling sectoral DFH (FSDFH)

The features of FDFH and SDFH are combined together into a new scheme named as fixed scheduling sectoral dynamic frequency hopping (FSDFH).The FSDFH exhibits the advantages of both, FDFH and SDFH without any additional overhead [9].

It is expected that FSDFH will decrease the chances of collisions in the overlapped areas. Hence, FSDFH will give the solution to the coexistence problem of WRAN systems [7]. 


\section{CONCLUSION}

IEEE 802.22 WRAN system is the first wireless communication standard based on cognitive radio functionalities. One of the major challenges for WRAN systems is known as coexistence problem. In this paper, we have presented an overview of WRAN and four schemes to reduce the coexistence problem in IEEE 802.22 WRAN, namely FDFH, SDFH, CDFH, and FSDFH.

\section{REFERENCES}

1) Lizdabel Morales-Tirado, Dr. Jeffrey H. Reed, advisor, "COGNITIVELY INSPIRED RADIO ENGINE FOR IEEE 802.22 WRAN", Mobile Portable Radio Group, Virginia Tech, Blacksburg, VA 24061.

2) "an overview or tutorial about IEEE 802.22, the Wireless Regional Area Network, WRAN standard using Cognitive Radio technology.", http://www.radio electronics.com/info/wireless/index.php.

3) Carlos Cordeiro, Kiran Challapali, Dagnachew Birru, and Sai Shankar N, "IEEE 802.22: The First Worldwide Wireless Standard based on Cognitive Radios".

4) Yun Hee Kim, "IEEE 802.22 WRAN Overview", School of Electronics and Information, Kyung Hee University, KOREA.

5) “IEEE 802.22", http://en.wikipedia.org/wiki/IEEE_802.22"

6) Kaigui Bian and Jung-Min "Jerry" Park, "A Coexistence-Aware Spectrum Sharing Protocol for WRANs", Department of Electrical and Computer Engineering Virginia Tech, Blacksburg, VA 24061.

7) Raed Al-Zubi, Mohammad Z. Siam, and Marwan Krunz, "Coexistence Problem in IEEE 802.22 Wireless Regional Area Networks", Department of Electrical and Computer Engineering University of Arizona, Tucson, AZ 85721.

8) W. Hu, D. Willkomm, L. Chu, M. Abusubaih, J. Gross, G. Vlantis, M. Gerla, and A. Wolisz, "Dynamic frequency hopping communities for efficient IEEE operation,” IEEE Communications Magazine, vol. 25, no. 5, pp. 80-87, May 2007.

9) "Cognitive Radio based IEEE 802.22 WRAN in the TV "White Space" Spectrum", http://amanuel-wran.blogspot.in/ 2012/06/challenges-of-wran.html. 